\title{
Cervical osteotomy for the correction of chin-on-chest deformity in ankylosing spondylitis
}

\author{
Technical note
}

\author{
Praveen V. Mummaneni, M.D., Valli P. Mummaneni, M.D., Regis W. Haid, JR., M.D., \\ Gerald E. RodTs, JR., M.D., AND Rick C. SASSo, M.D. \\ Department of Neurosurgery, The NeuroSpine Institute, and Department of Anesthesiology, \\ Emory University, Atlanta, Georgia; and Indiana Spine Group, Indianapolis, Indiana
}

\begin{abstract}
The correction of chin-on-chest deformity is challenging and requires combined anterior and posterior approaches to the cervical spine. The authors describe a cervical osteotomy technique for the correction of chin-on-chest deformity in patients with ankylosing spondylitis (AS). This procedure can be accomplished using a posterior screw rod construct combined with an anterior hybrid plate system.

In patients with AS, a "front-back-front" approach may be necessary because of the deformity's rigidity. The authors describe the complicated intubation and anesthetic requirements for this approach. They performed an anterior discectomy, cervical osteotomy, and unilateral pediculectomy but did not place anterior instrumentation. Via a posterior approach, laminectomies, facetectomies, and the contralateral pediculectomy were then undertaken. A posterior cervical screw/rod system was placed and loosely connected to titanium rods. Intraoperatively the deformity was corrected by placing the neck in extension combined with compression of the posterior screws on the rods. The posterior construct is then tightened. Finally, an anterior cervical approach is performed to place a structural interbody graft and a hybrid anterior cervical plate construct.

The authors have successfully used this approach to correct a chin-on-chest deformity in a patient with ankylosing spondylitis. At 1-year follow-up examination, excellent resolution of the deformity and solid fusion had been achieved. They prefer to perform this procedure by using state-of-the-art anterior and posterior instrumentation systems.
\end{abstract}

KEY WORDS - ankylosing spondylitis • osteotomy - kyphosis - cervical spine

The primary indication for cervical osteotomy is the correction of a fixed cervical kyphotic deformity, which occurs frequently in patients with AS. The deformity may affect horizontal gaze function, impede activities of daily living (such as swallowing), and induce disabling pain. A healed fracture in a case of AS or diffuse idiopathic skeletal hyperostosis may result in a fixed kyphotic deformity. In patients with AS kyphotic deformities may develop even in the absence of a traumatic fracture. In these patients bone occasionally becomes remodeled because of the weight of their own heads, and a slowly progressive cervical kyphotic deformity develops over a period of several years. ${ }^{3}$

Eventually, some of these patients develop a chin-onchest deformity that is not only painful but also limits their ability to eat and drink. In this end stage, these patients are unable to look straight ahead and try to compensate by

Abbreviations used in this paper: $\mathrm{AS}=$ ankylosing spondylitis; $\mathrm{MABP}=$ mean arterial blood pressure; $\mathrm{MEP}=$ motor evoked potential; $\mathrm{SCI}=$ spinal cord injury; SSEP $=$ somatosensory evoked potential; $\mathrm{VA}=$ vertebral artery; $\mathrm{VB}=$ vertebral body. flexing their hips and knees to reestablish sagittal balance (Sasso RC, Mummaneni VP, Mummaneni PV, in press).

Spinal osteotomy techniques for correction of cervical kyphosis have evolved steadily over the past three decades. In 1972, Simmons ${ }^{13}$ popularized the idea that cervical osteotomy could be performed in AS patients after administration of a local anesthetic and while using continuous neurological monitoring.

Simmons ${ }^{13}$ preferred to perform the osteotomy at C7-T1 for three reasons. First, the VA, which enters the foramen transversarium at $\mathrm{C}-6$, is avoided during the osteotomy. Second, the spinal canal at C7-T1 is significantly larger than in the midcervical spine, which decreases the possibility of SCI. Third, if catastrophic neurological injury were to occur during the osteotomy, damage at the level of C7-T1 as opposed to that at a midcervical level would allow the patient to retain upper-extremity function.

Simmons advocated a posterior wedge-shaped osteotomy by which to perform the correction. He removed the laminae and spinous processes from C-6 to T-1. Limited facetectomies were then conducted bilaterally to expose 
widely the C-8 nerve roots. The patient's head is extended to create an avulsion fracture of the anterior and middle columns of the ankylosed cervical spine with the instantaneous axis of rotation at the C-7 pedicle base. This causes lengthening of the anterior spine and shortening of the posterior elements. There is a risk of translation of the VB into the spinal canal during this maneuver, and SCI may result. Simmons did not use instrumentation; rather, halo immobilization was used until fusion occurred. This technical approach was associated with a $4 \%$ mortality rate and a $2 \%$ incidence of nerve root lesions. ${ }^{12}$

To limit the possibility of sagittal translation of the VB into the spinal canal during the aforementioned Simmons osteotomy, Mehdian, et al., ${ }^{7}$ devised a more controlled method of reduction at the osteotomy site. The Mehdian method involves the use of a posterior cervicothoracic screw/rod instrumentation system that is implanted prior to the completion of the osteotomy. Provisional, malleable rods are temporarily inserted. These rods bend, and the thoracic clamps slide along the rod as the reduction is performed without allowing translation. This allows for a more controlled reduction maneuver before the definitive titanium rods are placed.

We present our experience with the correction of cervical kyphotic deformity in a patient with AS; the procedures were performed using state-of-the-art instrumentation systems.

\section{CLINICAL MATERIAL AND METHODS}

\section{Preoperative Radiographic Evaluation}

Cervical kyphotic deformities can occur at any age and may be associated with thoracic and/or lumbar deformities. It is important, therefore, to obtain full-length posteroanterior and lateral 36-in scoliosis radiographs to examine overall sagittal and coronal balance in these patients. Such examination will ensure that the planned correction does not cause decompensation in overall spinal balance (Fig. 1). We assess cervical, thoracic, and lumbar sagittal alignment individually as well as globally and define the site of maximum kyphosis. The degree of required correction depends on the angle of the cervical deformity (the chin-brow to vertical angle). We determine the site of the osteotomy radiographically and cut out the posteriorly based wedge by using scissors and meticulously plan the procedure on the x-ray film, including notation of the position and size of screws. We estimate the needed deformity correction on the cut $\mathrm{x}$-ray film by approximating the screws above and below the site of the pedicle resection and then reassess the corrected sagittal balance. Such care is taken to avoid overcorrecting the kyphotic deformity.

We recommend preoperative evaluation by using CT myelography. The CT scan may be obtained using a STEALTH surgical navigation protocol (Medtronic Sofamor Danek, Memphis, TN) if such intraoperative guidance is available. Sagittal and coronal reconstructions are helpful to provide better visualization of the deformity. The CT scan is useful for measuring the dimensions of the VBs, the cervical pedicles, and the cervical lateral masses. This information is crucial for allowing proper selection of the interbody graft size and for selection of the appro-

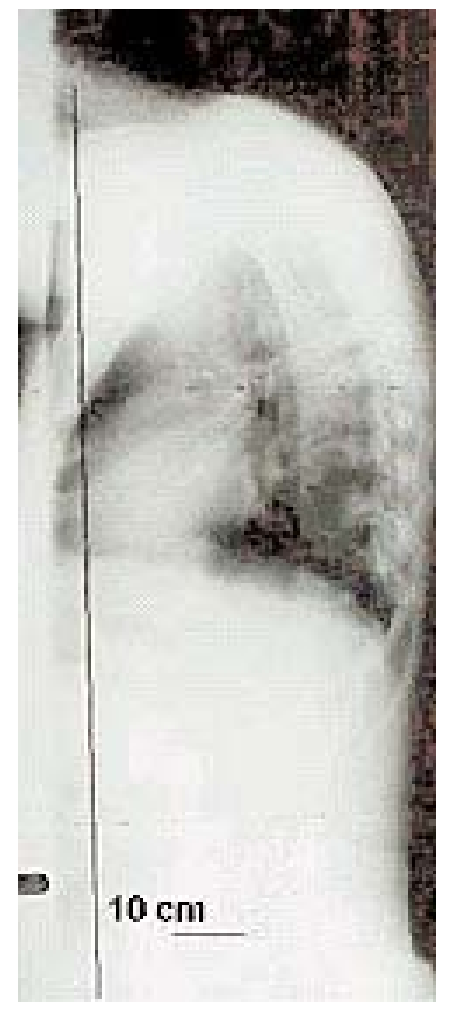

Fig. 1. Lateral 36-in standing radiograph demonstrating the sagittal plumb line $10 \mathrm{~cm}$ anterior to the sacral promontory in a patient with AS who sustained a C5-6 fracture 1 year previously. This healed causing significant fixed kyphosis at C-6.

priate screw lengths and widths for the cervicothoracic instrumentation. Myelography is useful for evaluating spinal cord and nerve root compression. Magnetic resonance angiography also may be performed to evaluate the size and position of the VAs.

\section{Operative Technique}

Anesthetic Considerations. Intubation of a patient with chin-on-chest deformity can be challenging. The potential exists for causing an SCI during intubation, and this potential is present throughout surgery because of hemodynamic changes in the spinal cord or direct cord injury. ${ }^{6}$

Consequently, modern anesthetic techniques involve monitoring the cardiovascular and neurological status. To this end, placement of an arterial line for blood pressure monitoring is recommended. Subclavian central line placement should be considered for monitoring the central venous pressure. Somatosensory evoked potential monitoring is recommended to ensure integrity of the spinal cord during the correction of the deformity. The use of MEP monitoring may also be considered.

We caution, however, that SSEP monitoring is not always accurate; there have been cases in which changes in evoked potentials have not been accompanied by changes in the patient's neurological status. There also have been cases in which a neurological injury has occurred without corresponding changes in the evoked potentials. 
One relatively safe method of intubation is awake, nasotracheal, fiberoptic intubation. Performing the intubation while the patient is awake is key to ensuring that an SCI does not occur during the intubation because neurological status can be continuously and easily assessed. The abnormal, fixed chin-brow angle does not allow for the patient's head to be extended, and fiberoptic intubation is necessary to visualize the vocal cords and ensure appropriate placement of the endotracheal tube.

Orotracheal intubation in chin-on-chest deformity is difficult at best because of restricted access to the oral cavity. Nasotracheal intubation is easier and can proceed after the nasal cavity has been anesthetized with an injection of $2 \%$ xylocaine and $0.25 \%$ phenylephrine.

The choice of anesthetic agents is critical when evoked potentials are monitored. Paralytics cannot be used in these cases because they blunt the MEPs. Likewise, nitrous cannot be used because it blunts SSEPs. One minimum alveolar concentration of vapor can also blunt evoked potentials. Consequently, we prefer to induce anesthesia by using propofol $(2-3 \mathrm{mg} / \mathrm{kg})$, and we maintain a propofol infusion throughout the case. Following induction, we prefer to use $50 \%$ of the minimum alveolar concentration of vapor (that is, isoflurane) and remifentanil $(0.1-0.25 \mu \mathrm{g} / \mathrm{kg} / \mathrm{min})$ as a narcotic infusion. This combination is least likely to affect the evoked potentials (Sasso RC, Mummaneni VP, Mummaneni PV, in press).

It is important to obtain another set of baseline readings of the evoked potentials once general anesthesia has been induced. Because alterations in the anesthetic regimen can cause changes in the evoked potentials, we try not to change the drugs or the doses throughout the case.

In addition, ensuring spinal cord perfusion throughout the case is important. We check the patient's MABP prior to intubation, and we maintain this MABP throughout the case (even when blood loss occurs). We do not hesitate to use transfusions and pressors (phenylephrine) as needed throughout the procedure to maintain the MABP and spinal cord perfusion.

Stage I: Anterior Release and Anterior Osteotomy. We obtained baseline readings of the SSEPs and the MEPs prior to initiating the anterior surgical stage. The patient was positioned supine on the operating table, and the head was rested on a foam headrest on top of folded blankets and pillows to adjust for the kyphotic angulation of the neck (Fig. 2). The shoulders were pulled caudally by using tape.

We prefer to perform the anterior cervical approach via a transverse left-sided incision for a C7-T1 kyphotic deformity to decrease the chance of injuring the recurrent laryngeal nerve. At higher levels, however, we prefer a right-sided approach. In this case, we used a right-sided approach to treat the C5-6 kyphosis.

After the standard anterior cervical exposure was performed, we placed distraction pins into the C-5 and C- 6 VBs and then obtained a localizing x-ray film to confirm the levels. The pins were placed perpendicular to the longitudinal axis of the VBs to help with orientation during the anterior resection (Fig. 3).

It is sometimes difficult to confirm levels in patients with AS because the disc spaces are fused. The surgeon, however, may confirm levels by counting the spinous pro-

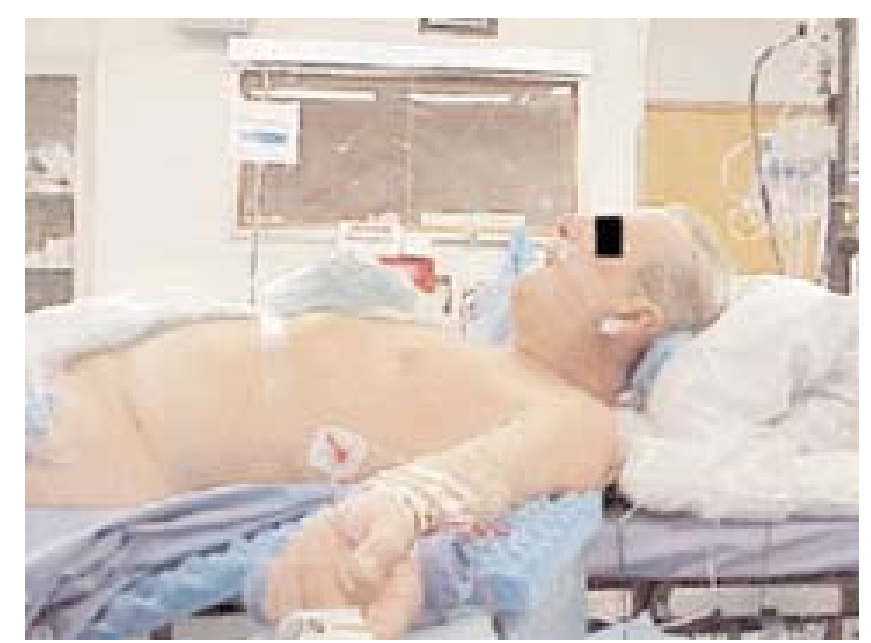

Fig. 2. Preoperative photograph of the patient positioned supine on the operating table. Pillows and towels stabilize the kyphotic head position. The anterior iliac crest bone graft incision is marked. Tricortical strut for the second anterior procedure is harvested as is cancellous bone for the posterior fusion.

cesses, by looking for the angulation in the cervical spine, or by assessing the location of the deformity in relation to the hyoid bone.

After localization of the appropriate levels, a generous discectomy was performed. Because the disc space was completely fused, a power drill was used. The posterior C-5 and C-6 endplates and all posterior vertebral osteophytes were removed. Posteriorly, a wedge of VB of C-5 and C- 6 was removed by undercutting the posterior VB walls.

After the generous wedge shape was created at C5-6, a left C-6 pediculectomy was performed. The left C-6 pedicle is accessible via a right-sided anterior cervical approach because the surgeon is looking across midline and can visualize the contralateral pedicle. The ipsilateral pedicle is difficult to visualize via the anterior approach, and the right pediculectomy was completed during the posterior stage of the operation.

Following the pediculectomy, the bilateral C-6 transverse foramina were exposed, and their bilateral anterior portions were removed (Fig. 4). This was done to prevent kinking of the VAs during the proceeding reduction maneuver.

Stage II: Posterior Release and Wedge Osteotomy. We carefully flipped the patient prone onto chest rolls on another operating table. The patient's head and shoulders were positioned off of the superior end of the table so that the Mayfield arm could be attached to the Mayfield head holder. We ensured that the patient's chin was not under pressure.

The plane of the patient's body was parallel to the floor. We noted the chin-brow angle and anticipated correcting this angle to allow the patient's face to be parallel to the floor at the completion of the posterior stage. Slight undercorrection of the angle is desirable as opposed to overcorrection because otherwise the patient is unable to see the ground while ambulating.

Following positioning, the evoked potentials were re- 


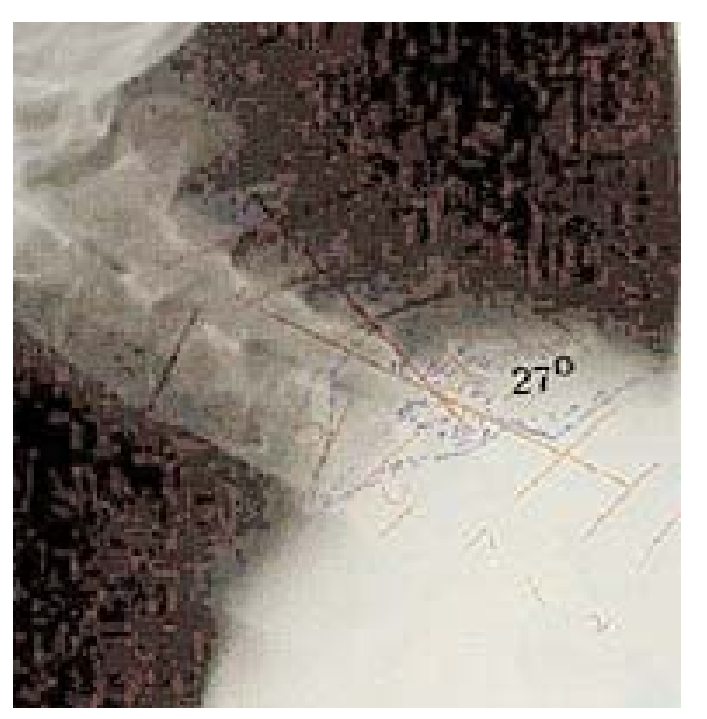

Fig. 3. Preoperative radiograph obtained to plan for circumferential osteotomy. The local kyphosis at C5-6 is $27^{\circ}$ due to previous fracture. Splaying of the C-6 posterior elements is present. A closing wedge osteotomy is planned with the base posterior and the hinge at the anterior C-5 VB. Lateral mass screws are planned to be placed into C-3, -4 , and -5 ; pedicle screws into C-7, T-1, and -2 .

checked to ensure the integrity of the spinal cord. We performed a standard posterior cervical exposure. Polyaxial screws were placed three levels above and three levels below the level of deformity (lateral mass screws at C-3 to C-5 and pedicle screws at C-7-T-2). Cervical lateral mass screws were placed using the trajectory and entry point, elucidated by members of our group, ${ }^{5}$ (Mummaneni PV, Sasso RC, Traynelis VC, in press) at the entry point $1 \mathrm{~mm}$ medial to the midpoint of the lateral mass and with trajectory aimed at the upper, outer quadrant of the lateral mass.

Placement of low cervical and upper thoracic pedicle screws are facilitated by standard anatomical landmarks; the craniocaudal entry point is at the junction of the upper and middle thirds of the transverse process, and this point is usually $3 \mathrm{~mm}$ below the facet joint itself. The mediolateral entry point is at the center of the facet joint. In addition, a laminoforaminotomy was performed to allow the surgeon to visualize and palpate the medial border of the pedicle $^{8}$ (Chun JY, Mummaneni PV, Birch BD, in press).

We used a high-speed drill to create a burr hole in the posterior cortex at the pedicle screw entry point. Typically, a bleeding cancellous channel (this is the cancellous bone within the pedicle) is exposed with this maneuver. We then used the tap from the VERTEX set (Medtronic Sofamor Danek, Memphis, TN) to enter the pedicle. This fine instrument usually "sinks" the pedicle down with minimal force. Its fine flutes are ideal for creating a path for the pedicle screw without perforating the pedicle walls. Cervical pedicle screws were then placed. ${ }^{9}$

Subsequently, we attached provisional rods to the C3-5 lateral mass screws and to the C7-T2 pedicle screws. These provisional rods are bowed out laterally and are attached to the polyaxial screw heads with small lateral connectors. The lateral attachment of the provisional rods was necessary to allow for the upcoming posterior wedge osteotomy of the cervical facets. Locking cap screws were placed and tightened to secure the connectors to the screw heads. Locking cap screws were also placed to secure the connectors to the rods, but we purposefully did not tighten these locking cap screws. Consequently, the rods could slide in the craniocaudal direction when the correction was performed.

We then performed the posterior wedge osteotomy. First, the inferior half of the C-5 spinous process and lamina were removed. If this bone is not removed, it will impinge on the posterior dura mater when the correction is performed. The inferior articulating C-5 facet was resected below the C-5 lateral mass screw. Next, all of the C-6 posterior elements were removed, including the spinous process, lamina, superior articular process, inferior articular process, and the residual C-6 pedicles (the portion of the C-6 pedicles that was not removed during the anterior approach). The exiting C-6 nerve roots were completely exposed when the removal of all osseous material was completed.

We removed the posterior portion of the transverse foramen to complete a bilateral foramen transversectomy at C-6. This was necessary to prevent kinking of the VA during the correction. There was abundant perivertebral venous plexus bleeding at this stage, which was controlled with thrombin-soaked gelfoam.

Finally, the cephalad half of the C-7 spinous process and lamina were removed, also to prevent impingement on the posterior dura during the correction. The superior articulating facet of C-7 cranial to the C-7 pedicle screw was also removed (Fig. 5 left).

We were then ready to perform the correction. The instantaneous axis of rotation for the shortening/closing osteotomy was the anterior C-5 VB. One surgeon left the operating field and went to the Mayfield headrest to change the chin-brow angle. Evoked potentials were recorded before and during this maneuver. If changes are observed during the correction, the procedure is suspended until the evoked potentials return to baseline.

The unscrubbed surgeon then detached the Mayfield arm from the head holder and slowly extended the head until the patient's face was parallel to the floor. Meanwhile, during this pivotal portion of the procedure, the scrubbed surgeon closely monitored changes in the dura, nerve roots, and the position of the instrumentation. The residual C-5 and C-7 posterior elements were closely

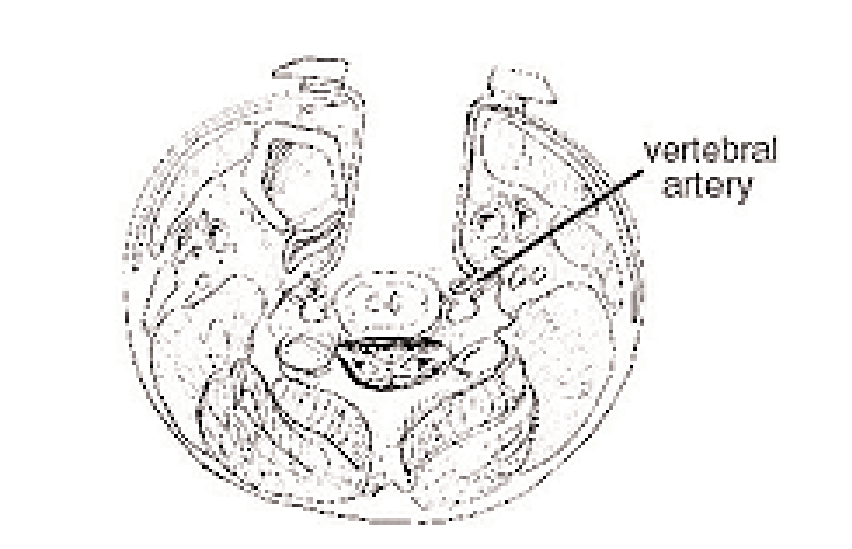

Fig. 4. The anterior portions of the transverse foramina are removed to release the VBs at C-6. 


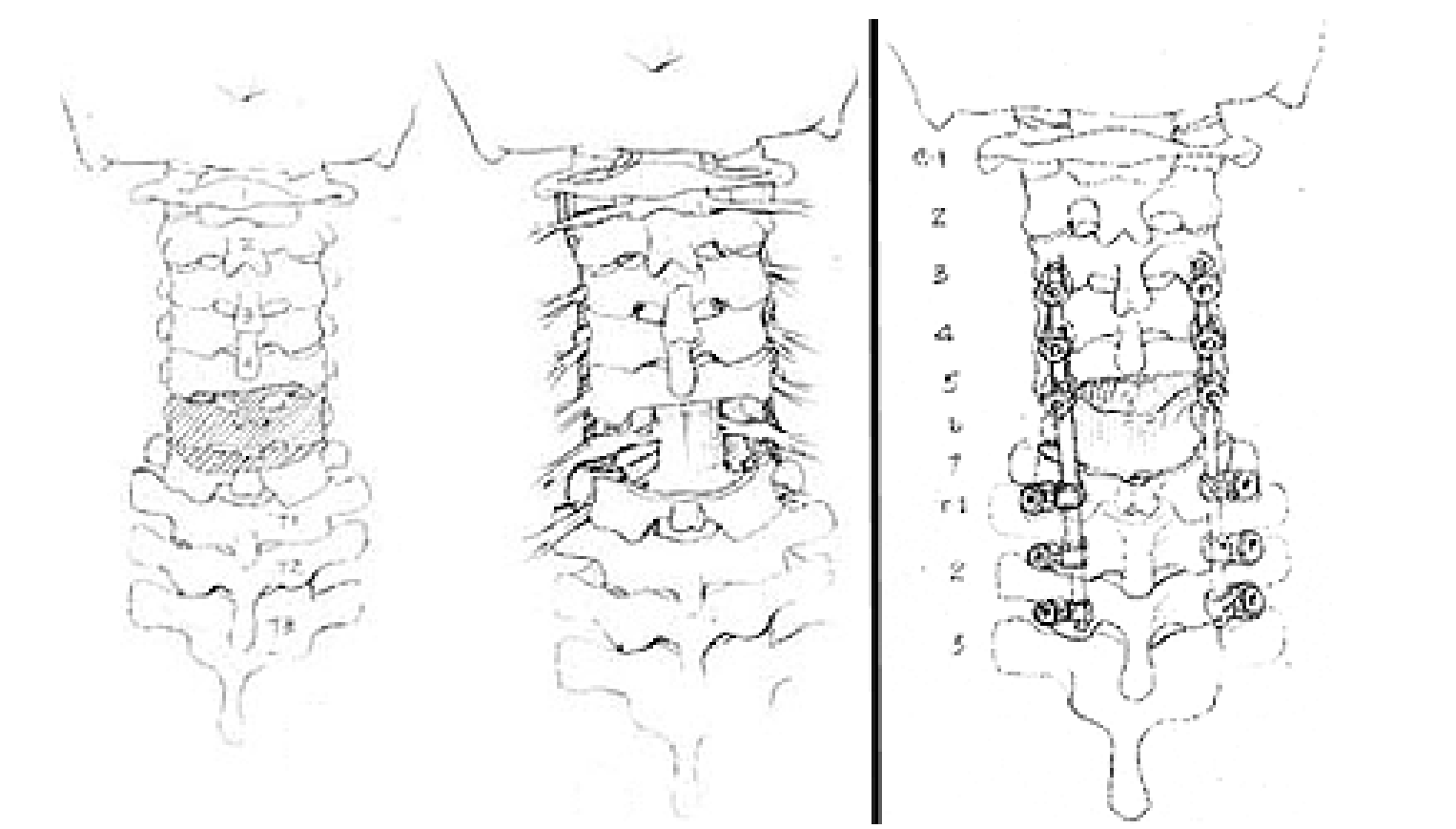

Fig. 5. Aspects of the posterior procedure. Left: Appearance of the spine after resection. Posterior resection. Right: Definitive posterior rods in place.

monitored to ensure that they did not impinge on the posterior dura as the neck was extended. The connectors slid along the provisional rods as the correction was performed; these rods were malleable and bent as the correction was performed. The provisional rods prevent sudden translational movements during the correction, allowing the surgeons to perform a safe, controlled correction (unlike that performed by Simmons $\left.{ }^{12,13}\right)$. During the correction, the posterior dura typically relaxes and mild infolding may occur.

When the unscrubbed surgeon completed the extension and the patient's chin-brow angle returned to the normal position, he locked the Mayfield head holder into the new position. Evoked potentials were again checked to ensure that the extension maneuver did not cause SCI. The scrubbed surgeon then measured, cut, and contoured the permanent titanium rods by using the provisional rods as templates. The provisional rods were then replaced by the permanent rods, which were secured to the screw heads to maintain the corrected position (Fig. 5 right).

The final steps in the second stage involved decorticating C3-5 and C7-T2 regions by using a high-speed drill. We delayed the decortication until the end of Stage II to minimize bleeding. The cancellous autograft harvested from the iliac crest was then placed over the decorticated areas to create a fusion. A standard layered closure was performed.

Stage III: Anterior Instrumentation. The patient was then flipped supine onto the other operating table, after which evoked potentials were again checked. The head was placed on a foam cushion and the Mayfield pins were removed. The anterior incision was reopened, distraction pins were replaced into the C-5 and C- 6 VBs, and mild distraction was performed. A tricortical iliac autograft was placed into the now-parallel C5-6 interbody space. The distraction pins were removed, and an Atlantis anterior cervical plate (Medtronic Sofamor Danek) was placed (Fig. 6 upper).

We prefer to use a hybrid construct with the shortest possible Atlantis plate to prevent adjacent-level degeneration. The Atlantis hybrid system consists of two fixed-

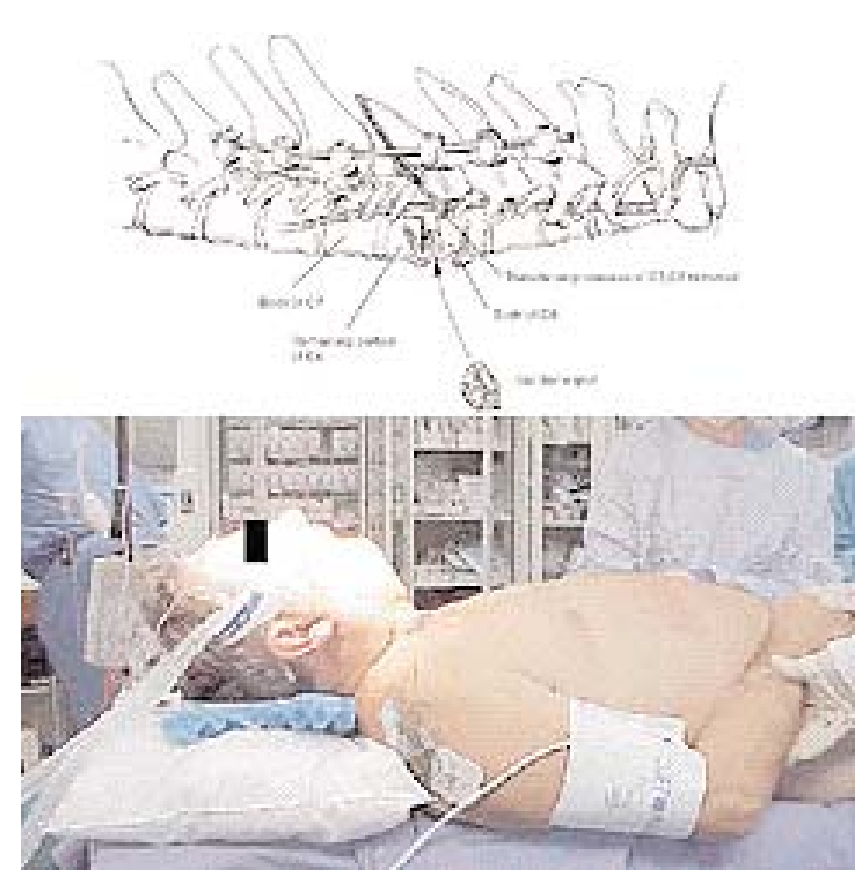

Fig. 6. Upper: Final construct in place and reduction of kyphotic deformity. Lower: Photograph obtained immediately after the third stage. Note the significant correction of kyphosis compared with that in Fig. 2. 


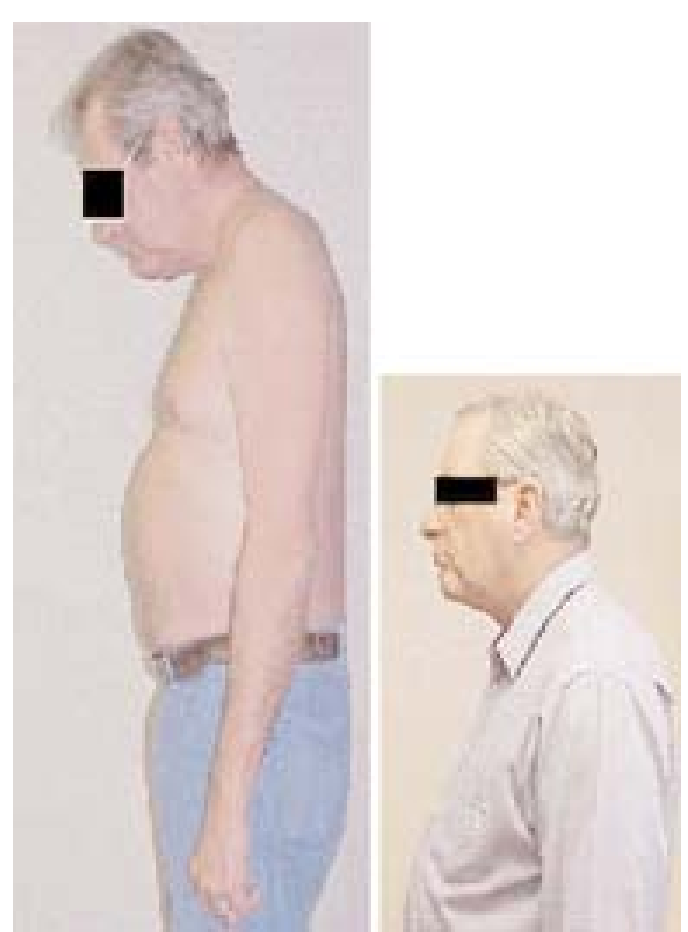

Fig. 7. Preoperative (left) and postoperative (right) photographs showing the patient in this case.

angle screws at C-6 and two variable-angle screws at C-5. The lower fixed screws act as a "buttress" while the upper screws allow for graft subsidence. ${ }^{1,4,10}$ An anterior drain was placed, and the anterior cervical skin incision was closed with subcuticular suture (Fig. 6 lower) A Miami J collar was placed.

\section{Postoperative Management}

The patient was kept intubated and taken to the recovery room; postoperatively, he was allowed to temporarily awaken from the anesthesia to engage in a neurological examination. He was extubated after the anesthesiologist assessed airway edema with a leak test. Progressive ambulation was initiated immediately and the cervical collar was kept in place for 6 weeks.

At 1 year follow up, the spinal correction was maintained and a solid fusion had occurred. He was neurologically intact.

\section{CONCLUSIONS}

Cases of chin-on-chest deformity pose an anesthetic and surgical challenge. Modern anesthetic and surgical techniques allow for a relatively safe correction of this deformity (Fig. 7). Provisional rods used with the VERTEX system allow for controlled reduction of the defor- mity after the osteotomy has been performed. Our technique is not the only method of achieving reduction of this deformity, but it is one relatively safe option in cases of this rigid deformity in patients with $\mathrm{AS}^{2,11}$

\section{Acknowledgments}

The authors are grateful to Sherry Ballenger, Regina Carder, and Drew Imhulse for assistance with the manuscript.

\section{References}

1. Barnes B, Haid RW, Rodts G, et al: Early results using the Atlantis anterior cervical plate system. Neurosurg Focus 12(1): Article 13, 2002

2. Duff SE, Grundy PL, Gill SS: New approach to cervical flexion deformity in ankylosing spondylitis. Case report. J Neurosurg (Spine 2) 93:283-286

3. Gallager M, Haid RW: Spondyloarthropathies including ankylosing spondylitis, in Youmans JR (ed): Neurological Surgery: A Comprehensive Reference Guide to the Diagnosis and Management of Neurosurgical Problems, ed 4. Philadelphia: WB Saunders, 1996, pp 2262-2283

4. Haid RW, Foley KT, Rodts GE, et al: The Cervical Spine Study Group anterior cervical plate nomenclature. Neurosurg Focus 12 (1):Article 15, 2002

5. Haid RW, Papadopoulos SM, Sonntag VKH: Lateral mass plating for cervical instability. J Neurosurg 74:342, 1991 (Abstract)

6. Kamarkar US, Chaudhari LS, Hosalkar H, et al: Difficult intubation in a case of ankylosing spondylitis: a case report. J Postgrad Med 44:43-46, 1998

7. Mehdian SM, Freeman BJ, Licina P: Cervical osteotomy for ankylosing spondylitis: an innovative variation on an existing technique. Eur Spine J 8:505-509, 1999

8. Mummaneni PV, Haid RW, Traynelis VC, et al: Posterior cervical fixation using a new polyaxial screw and rod system: technique and surgical results. Neurosurg Focus 12(1): Article 8, 2002

9. Mummaneni PV, Ondra SL, Haid RW: Principles of spinal deformity-Part 2: operative management. Contemp Neurosurg 24:1-9, 2002

10. Mummaneni PV, Srinivasan JK, Haid RW, et al: Overview of anterior cervical plating. Spinal Surgery 16:207-216, 2002

11. Schultz KD Jr, McLaughlin MR, Haid RW Jr, et al: Singlestage anterior-posterior decompression and stabilization for complex cervical spine disorders. J Neurosurg (Spine 2) 93: 214-221, 2000

12. Simmons EH: Kyphotic deformity of the spine in ankylosing spondylitis. Clin Orthop 128:65-77, 1977

13. Simmons EH: The surgical correction of flexion deformity of the cervical spine in ankylosing spondylitis. Clin Orthop 86: 132-143, 1972

Manuscript received December 6, 2002.

Accepted in final form December 23, 2002.

Address reprint requests to: Praveen V. Mummaneni, M.D., Department of Neurosurgery, Emory University, Crawford Long Hospital, 550 Peachtree Street, Suite 806, Atlanta, Georgia 30308. email: praveen_mummaneni@emoryhealthcare.org. 